\title{
A Practical Guide to Interpreting FDG PET and CT Nodal Findings in Lung Cancer
}

Fatemeh Behnia $^{1 *}$, Antoine Leblond ${ }^{2}$ and Hubert Vesselle ${ }^{1 *}$

${ }^{1}$ Department of Radiology, Division of Nuclear Medicine, University of Washington, Seattle, WA, USA

${ }^{2}$ University of Montreal, University of Montreal Hospital Center, St-Dominique, Montreal, QC, Canada

\begin{abstract}
FDG PET/CT has been used to help characterize indeterminate pulmonary nodules, and to stage Non-Small Cell Lung Cancer (NSCLC) since January of 1998. The ever-expanding utilization of this modality in oncologic imaging cancer patients presented unique challenges for imagers who may not have received specialized training in the interpretation of PET/CT images. Interpretation of FDG PET/CT is particularly challenging in the thorax where inflammatory processes are common and often coexist with malignancy, rendering accurate tumor staging difficult. This article presents a systematic approach to FDG PET/CT interpretation in lung cancer staging, addressing common confounding situations often encountered. Although this illustrative discussion focuses on lung cancer staging, most of these tips could be applied to other types of cancer as well.
\end{abstract}

Keywords: Pulmonary nodules; Non-small cell lung cancer; FDG PET/CT scan

\section{Commentary}

Lung cancer staging is a key to select appropriate treatment and predicting prognosis. The most widely used staging system is the TNM system, which uses the size of the primary tumor (T), the extent of nodal involvement $(\mathrm{N})$, and presence or absence of distant metastases $(\mathrm{M})$ [1]. Treatment of choice for lung cancer is surgical resection when possible. While early stage lung cancers (stages I and II) undergo primary resection, stages IIA and IIB benefit from adjuvant chemotherapy prior to resection to improve long-term survival. Stage IIIA-N2 patients usually undergo surgery following neoadjuvant chemotherapy with or without concurrent radiotherapy. Stage IIIB patients, due to T4 or $\mathrm{N} 3$ status, are not considered to be surgical candidates and will receive definitive chemoradiotherapy. Stage IV lung cancers and those with malignant pleural effusions are treated with chemotherapy only [2]

CT is the initial imaging modality for diagnosis and is accurate for measurement and assessment of the primary tumor $(\mathrm{T})$. It may reveal distant metastases (M status). For nodal staging, CT mainly relies on nodal size. Size cut-off has a limited accuracy for staging lung cancer. Accuracy of CT for mediastinal nodal staging is, therefore, low [3-7].

Lungs are a portal-of-entry for many environmental inflammatory and infectious agents. Inflammatory nodes may be enlarged on CT, as well as hypermetabolic on PET. While implementing an SUV cut-off, as suggested by some investigators, might increase negative predictive value, it does not increase accuracy as degree of FDG uptake between inflammatory and malignant nodes greatly overlap [8]. Diagnostic performance of PET-CT particularly suffers when inflammatory lung disease and lung cancer co-exist (Figure 1) [9].

The FDG PET/CT image set is essential for interpretation of these nodes. An FDG PET/CT examination should consist of:

\section{A non-attenuation corrected FDG PET image volume (NAC)}

2. An attenuation-corrected FDG PET image set scaled to the injected dose of $18 \mathrm{~F}-\mathrm{FDG}$ and to patient's body weight to generate Standard Uptake Value (SUV)

\section{A CT image set.}

A systematic approach should include evaluation of all of these images, starting with the NAC images, followed by the SUV and CT images.

As with any imaging study, the first step required is to check the quality of the images and of the reconstructions. Evidence of poor fasting, recent insulin administration or misregistration between SUV and NAC images should be identified. Image noise and texture need to be appreciated as they may affect the sensitivity and specificity of the interpretation.

Interpretation of FDG PET includes detection and characterization tasks. This manuscript aims at integration of FDG PET and CT findings to help improve characterization of nodal uptake in lung cancer.

Initial evaluation of a FDG PET/CT image set is best made without any knowledge of the patient's medical history, so that the initial approach to images will be unbiased. The final interpretation, however, requires thorough knowledge and integration of all clinical history, and all prior imaging available.

Important to the staging of any tumor type is knowledge of lymphatic drainage for each region of the body. In the thorax, lymph flows centripetally from the lungs to the hila and mediastinum, resulting in well described patterns of malignant adenopathy. Right upper lobe (RUL) tumors spread to the superior right hilum and then to the right tracheobronchial angle and right paratracheal stations. It is also possible for a right upper lobe lesion to directly metastasize to right paratracheal nodal station. Likewise, left upper lobe (LUL) tumors can spread to the left hilum, with the additional possibility of direct spread to the aorto-pulmonary (AP) window nodes. Rarely

*Corresponding author: Hubert Vesselle, Department of Radiology, Division of Nuclear Medicine, University of Washington, 1959 N.E. Pacific Street, Box 357115, Seattle, WA-98195, USA, Tel: 206-616-5781; Fax: 206-543-6317; E-mail: vesselle@uw.edu

Received July 21, 2016; Accepted November 24, 2016; Published November 30, 2016

Citation: Behnia F, Leblond A, Vesselle H (2016) A Practical Guide to Interpreting FDG PET and CT Nodal Findings in Lung Cancer. J Nucl Med Radiat Ther 8: 319. doi: 10.4172/2155-9619.1000319

Copyright: $\odot 2016$ Behnia F, et al. This is an open-access article distributed under the terms of the Creative Commons Attribution License, which permits unrestricted use, distribution, and reproduction in any medium, provided the original author and source are credited. 
Citation: Behnia F, Leblond A, Vesselle H (2016) A Practical Guide to Interpreting FDG PET and CT Nodal Findings in Lung Cancer. J Nucl Med Radiat Ther 8: 319. doi: 10.4172/2155-9619.1000319

Page 2 of 5

upper lobe tumors such as Pancoast tumors will spread directly to the adjacent supraclavicular nodal station. Right middle lobe tumors spread to the right hilum. Lower lobe tumors will generally spread to the inferior hilum and/or the subcarinal station. Lower lobe tumors can also spread to pulmonary ligament nodes. Left lower lobe cancers have the additional option of giving rise to malignant adenopathy in the para-esophageal station. Only when ipsilateral mediastinal spread has occurred contralateral spread is enabled [10].

Combining FDG PET and CT has been shown to increase diagnostic accuracy in mediastinal staging of non-small cell cancer (NSCLC) patients [11]. The SUV by itself cannot be relied upon for differentiating malignant from benign lymph nodes $[4,12]$ however, relating intensity of uptake in the node as compared to primary tumor or uptake per unit volume, could increase accuracy of interpretation. This is explained further in the schematic illustrations that follow (Figure 2).

In scenario $2 \mathrm{~A}$, the node is $2 \mathrm{~cm}$ is short axis, compared to $0.8 \mathrm{~cm}$ in scenario $2 \mathrm{~B}$. In which scenario is the node most likely malignant? The smaller node of case $2 \mathrm{~B}$ is more likely malignant because its uptake per unit volume is more commensurate with that of the primary tumor. If cancer cells of the primary had populated the enlarged node of case $2 \mathrm{~A}$, its SUV would have been expected to be higher than 3.5 , and closer to that of the primary. This example illustrates that the uptake per unit volume of a node has to be related to that of the primary tumor in order to determine its etiology.

In Figure 3A two of these nodes (right hilar and right paratracheal stations) have FDG uptake with SUV of 3.5, the remainder of nodes are not FDG avid. In Figure 3B, all nodes have similar SUV of 3.5. Nodes shown on Figure 3A, are at the expected drainage site for the RUL primary. Also, their uptake per volume is comparable to that of the primary tumor. The location of nodes in Figure 3B does not correlate to the drainage path expected from a RUL primary. Furthermore, SUV and size of malignant nodes are expected to decrease with distance away from the primary tumor along the lymphatic drainage pathway. This is a benign pattern of fairly uniform uptake in the setting of inflammation and/or infection. We call this a "buckshot pattern", frequently associated with sarcoidosis as well as inflammatory lung disease. In Figure 3A, there is a combination of reactive non-FDG avid adenopathy and superimposed malignant adenopathy at right hilum and right tracheobronchial angle stations (Figure 4-7).

If a node stands out in uptake over the background "buckshot" inflammatory adenopathy pattern, it must be located in a nodal station draining the primary tumor before being considered malignant and related to the primary lung cancer. Figure 7A demonstrate $\mathrm{s}$ RUL nodule with SUV of 10 . Several hilar and mediastinal nodes
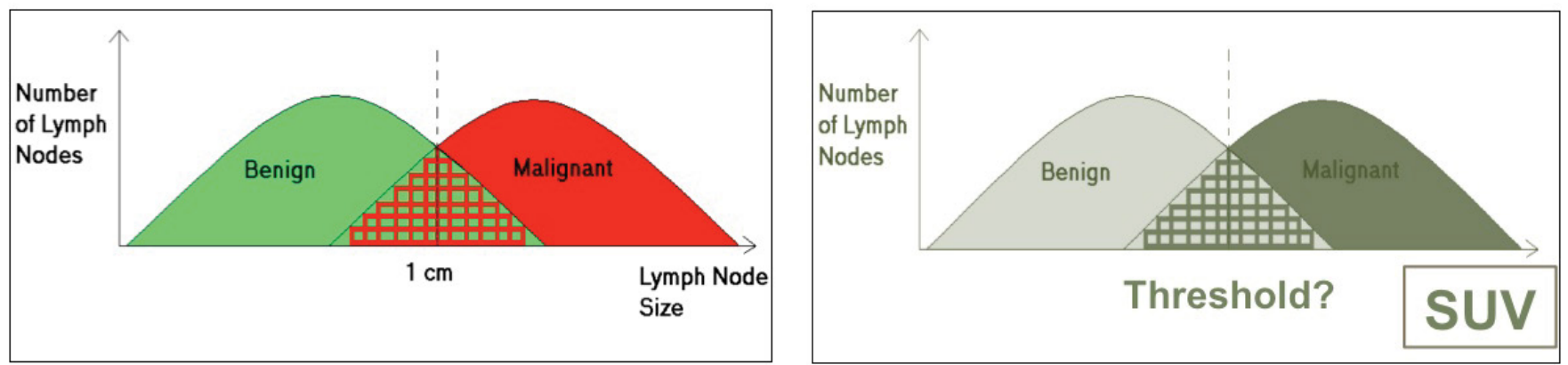

Figure 1: Histograms of benign and malignant nodes based on size (left) and SUV (right). Both size cut-off and SUV threshold are not specific.

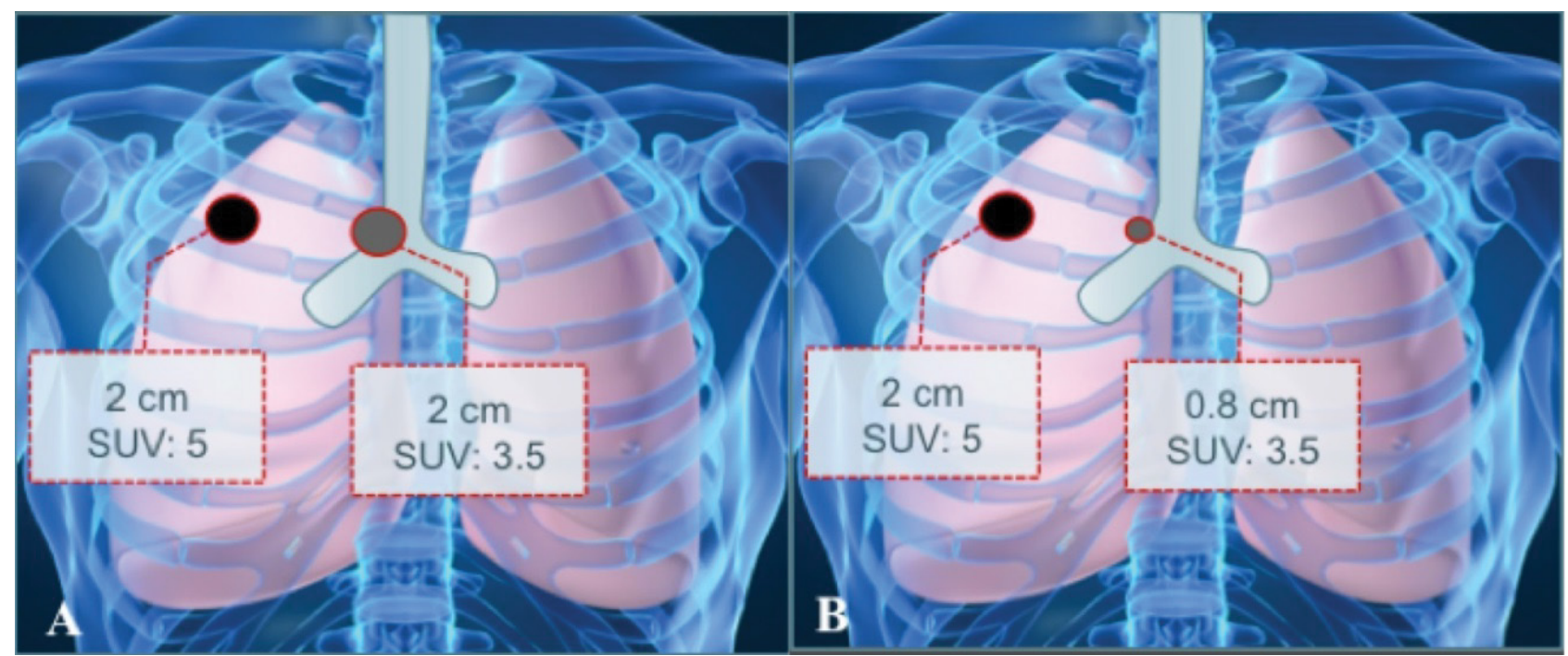

Figure 2: A and B show a $2 \mathrm{~cm}$ RUL primary tumor with SUV of 5, and a right tracheobronchial node with SUV of 3.5 . The lymph node measures $2 \mathrm{~cm}$ in short axis on $A$ and $0.8 \mathrm{~cm}$ on $B$. Note that uptake per unit volume of the smaller node is higher and is more commensurate with that of the primary tumor. This node is therefore, more likely to be malignant. 
Citation: Behnia F, Leblond A, Vesselle H (2016) A Practical Guide to Interpreting FDG PET and CT Nodal Findings in Lung Cancer. J Nucl Med Radiat Ther 8: 319. doi: 10.4172/2155-9619.1000319
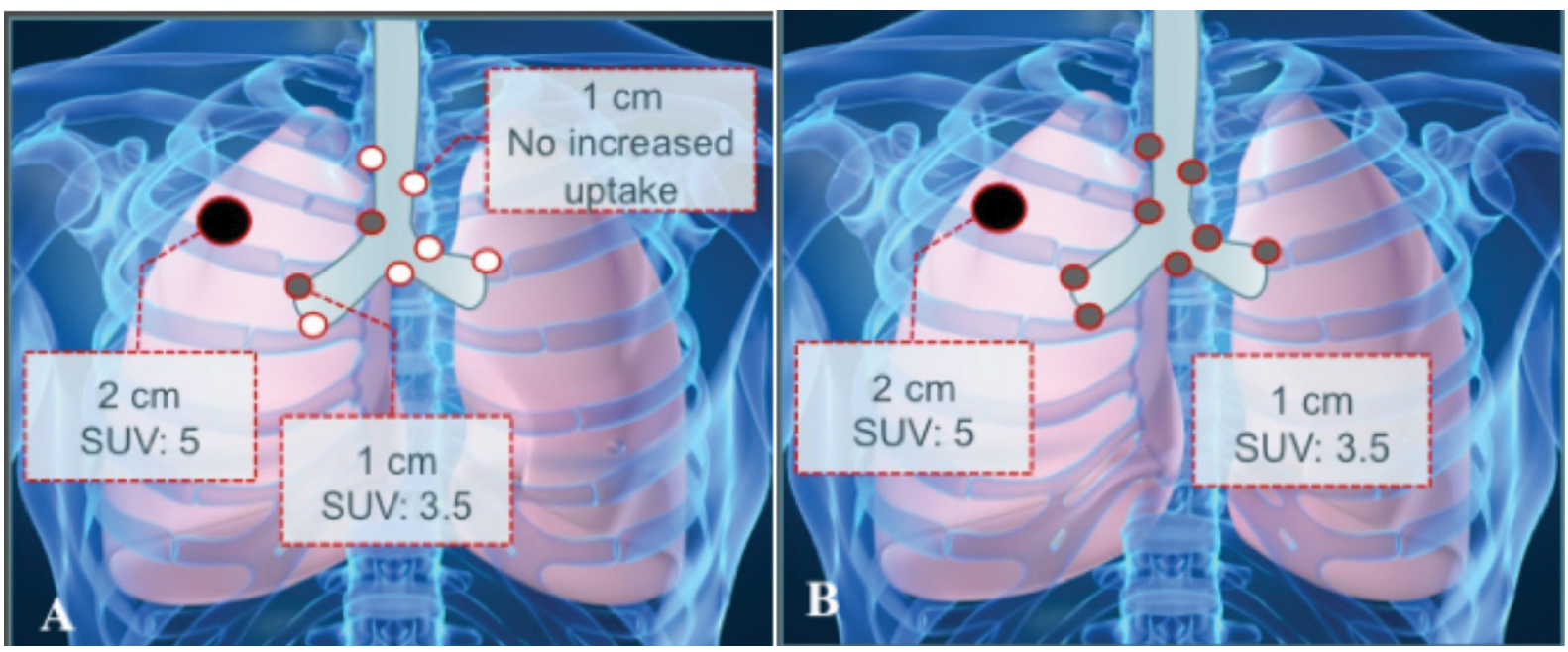

Figure 3: Bilateral hilar and mediastinal nodes of similar size of $1 \mathrm{~cm}$. Right hilar and paratracheal nodes in A have SUV of 3.5, while remainder of the nodes are not FDG avid. Moreover, the two hypermetabolic nodes are in the expected lymphatic drainage pathway for the primary RUL tumor. These nodes are likely malignant. Although all nodes on B are FDG avid, uniformity of size and SUV makes them more likely to be inflammatory. Also, SUV and size of metastatic nodes would decrease with distance away from the primary.

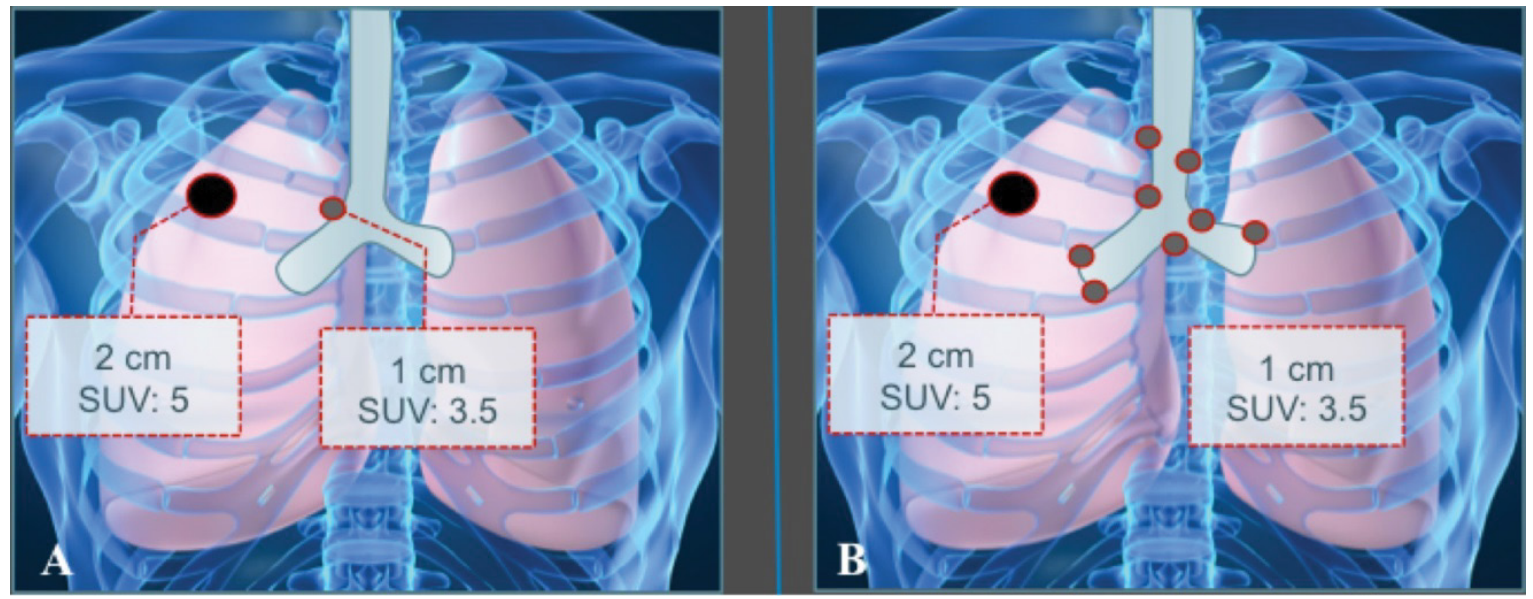

Figure 4: Another example of a malignant right tracheobronchial node (A), versus a benign inflammatory "buckshot" pattern (B).

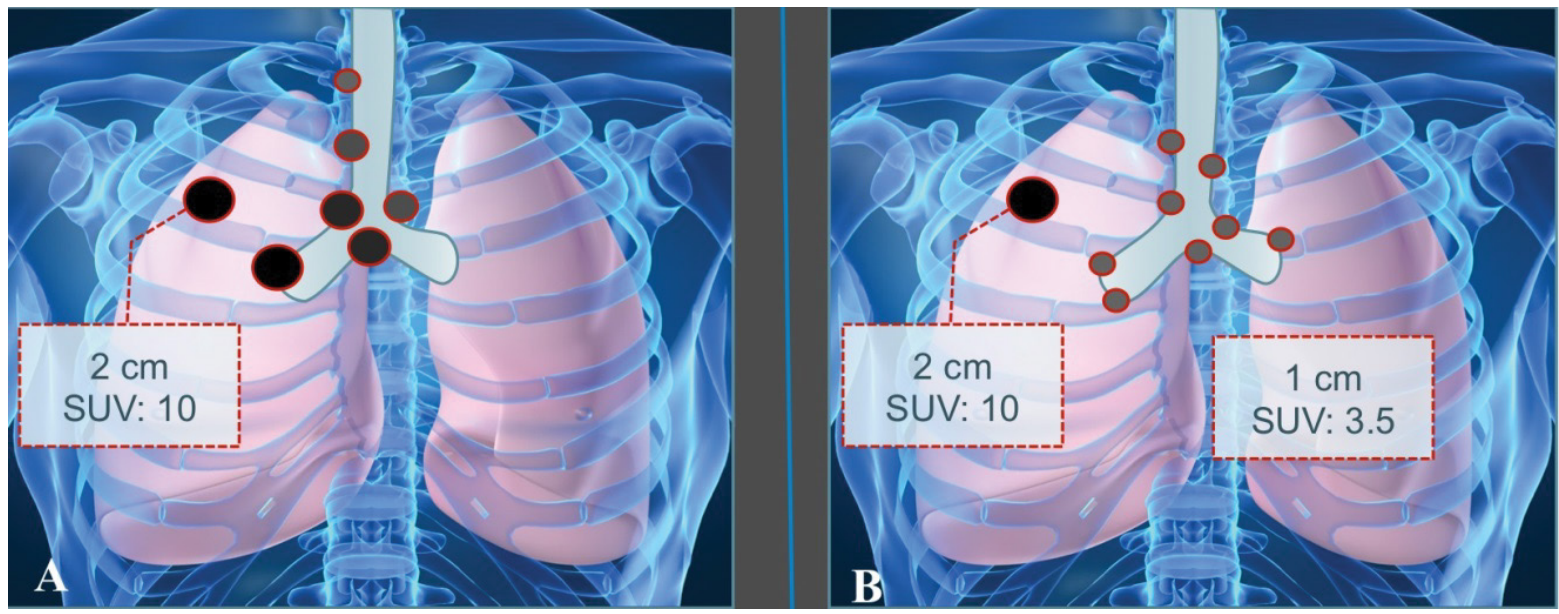

Figure 5: Expected pattern with primary cancer in RUL involving right hilar and bilateral mediastinal nodes. Note that size and SUV of metastatic nodes decrease with distance away from the primary tumor (A). Compare with the "buckshot pattern" of inflammation (B). 
Citation: Behnia F, Leblond A, Vesselle H (2016) A Practical Guide to Interpreting FDG PET and CT Nodal Findings in Lung Cancer. J Nucl Med Radiat Ther 8: 319. doi: 10.4172/2155-9619.1000319

Page 4 of 5

demonstrate similar size and uptake consistent with an inflammatory process. Figure 7B shows a similar RUL nodule. There is, however, a left paratracheal node that demonstrates higher SUV than the other hilar and mediastinal nodes. Based on surgical series, the contralateral left paratracheal node is not related to the RUL lung cancer since malignant adenopathy would not be expected to spare ipsilateral nodal stations to involve a contralateral station. The high uptake per unit volume does raise concern for malignancy, which could be originating from a separate left lung primary cancer or may represent another unrelated malignant process (lymphoma, etc.).

This is a companion illustration to Figure 2. In Figure 8A, the primary tumor is very FDG avid per unit volume. If the node is FDG avid because cancer cells populate it, it should have a much higher SUV value given that its size is near identical to that of the primary tumor. In Figure 8B, the node has uptake for size that is in keeping with that of the primary tumor making it likely to be malignant. Hence, the same node can be malignant in one setting and not in another. In other words, no SUV threshold would have enabled one to properly identify the malignant node in this case. This illustration underscores again that the uptake per unit volume of the node has to be related to that of the primary tumor in order to assess the likelihood of a node being malignant.

On the left, Figure 9A, a small RUL nodule with SUV of 8 is seen. The node is unlikely to be malignant because it is of the same size as the primary tumor but much lower SUV. On Figure 9B, the primary again has an SUV of 8 , but is larger. As a result, the nodal uptake per unit volume is more in keeping with that of the primary tumor. The node on Figure 9B is, therefore, more likely to be malignant. For the node in figure $8 \mathrm{~A}$ to be malignant, a higher SUV would have been expected.

\section{Conclusion}

Because the lungs provide a portal of entry for inflammatory or infectious agents, it is common for multiple prominent nodes to be present on chest CT and demonstrate some FDG avidity at FDG PET. The uniform pattern of uptake per size for multiple nodes across both hila and bilateral mediastinum should be recognized as a typical pattern of inflammatory adenopathy at FDG PET/CT, or "buckshot" pattern. This common pattern of inflammatory adenopathy often co-exists with lung cancer rendering its accurate nodal staging very difficult with CT only. The nodal staging accuracy of FDG PET is affected less by inflammatory adenopathy than that of CT, optimal staging accuracy

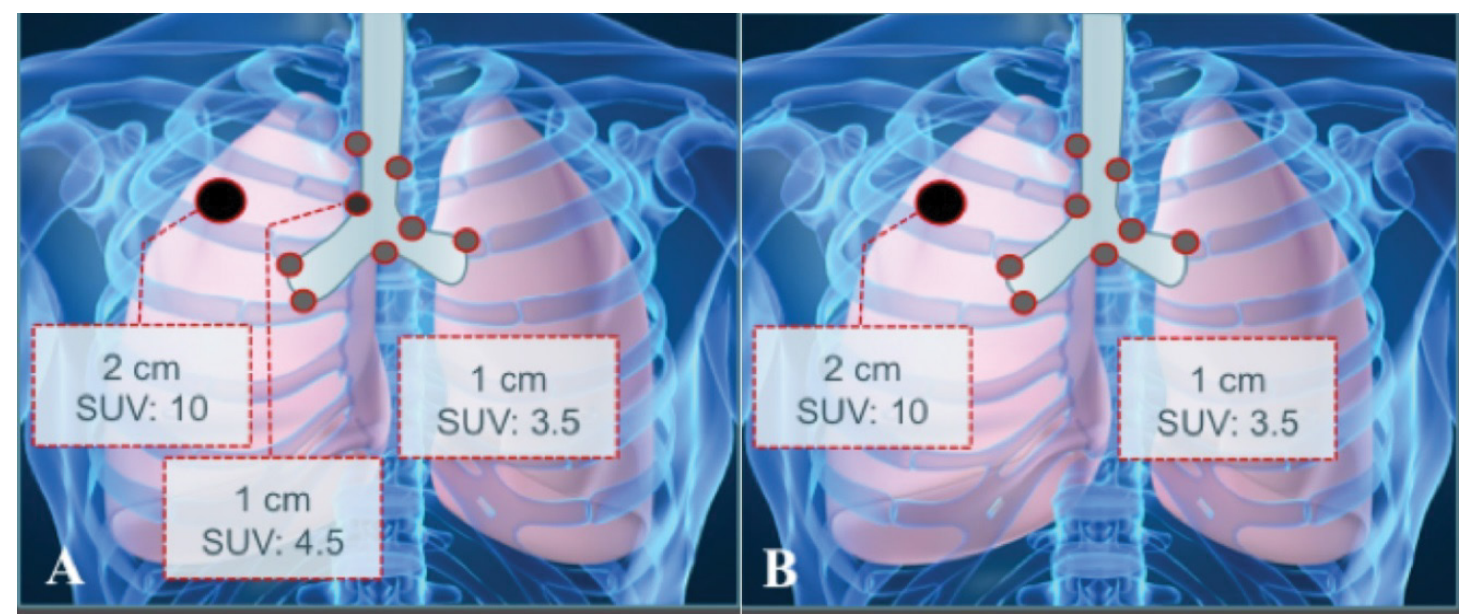

Figure 6: $2 \mathrm{~cm}$ RUL tumor with SUV of 10. Compare metastatic right tracheobronchial node in a background of inflammatory lung disease (A), with "buckshot pattern" of inflammatory process $(B)$.
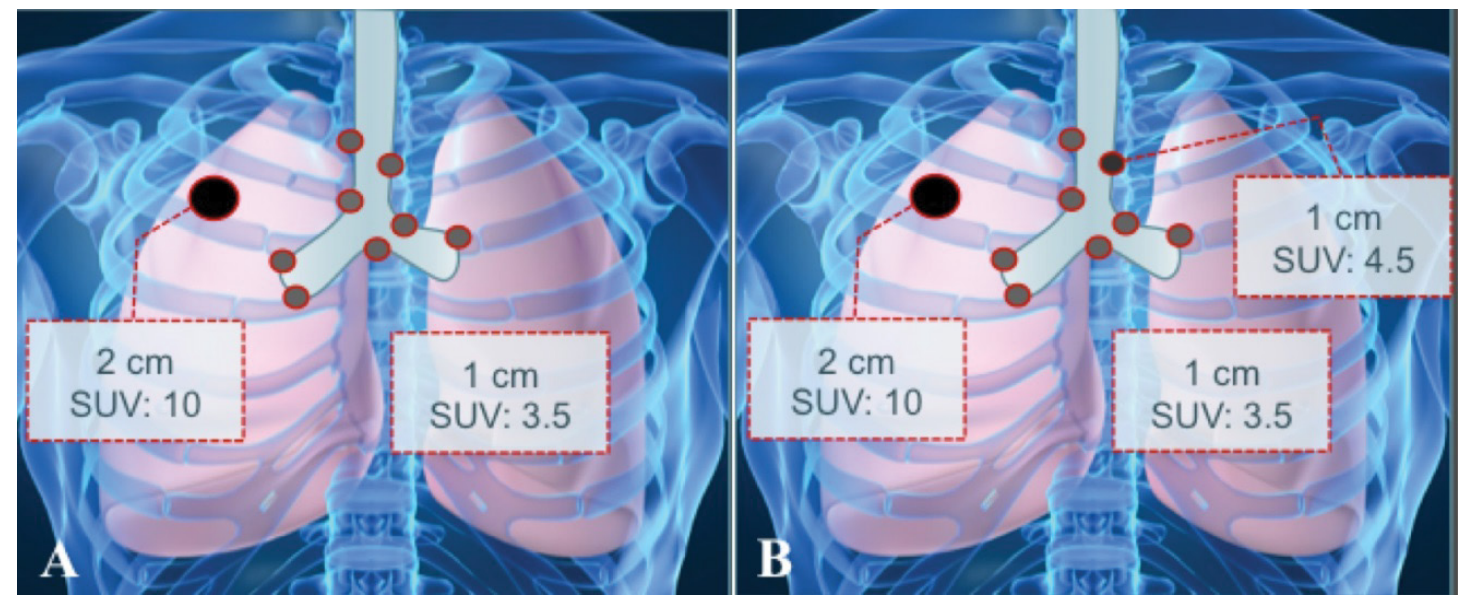

Figure 7: Besides SUV, location of a node has to relate to lymphatic drainage pattern. On A, nodes are likely inflammatory given uniform size and uptake. Contralateral mediastinal node on B, despite higher SUV than the rest of hypermetabolic nodes, cannot represent metastasis from a RUL tumor. 
Citation: Behnia F, Leblond A, Vesselle H (2016) A Practical Guide to Interpreting FDG PET and CT Nodal Findings in Lung Cancer. J Nucl Med Radiat Ther 8: 319. doi: 10.4172/2155-9619.1000319
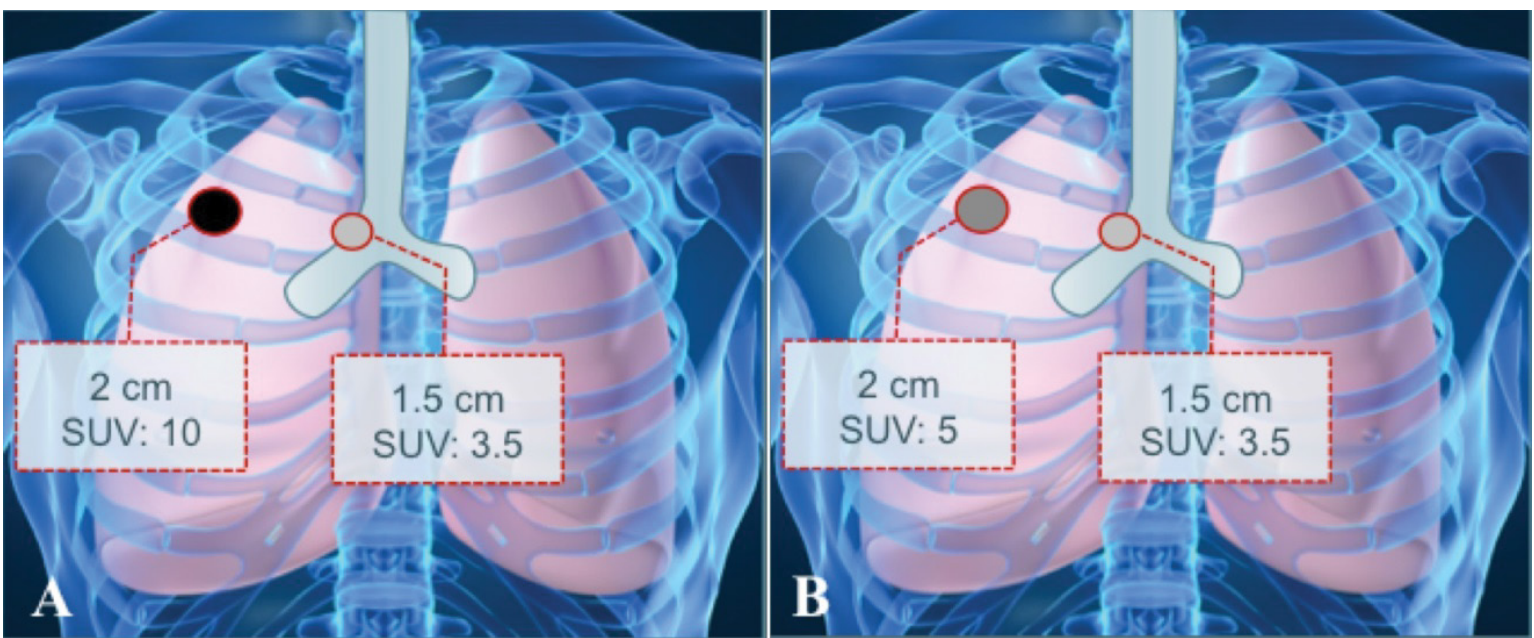

Figure 8: A) the primary tumor is very FDG avid per unit volume. B) the node has uptake for size that is in keeping with that of the primary tumor making it likely to be malignant.

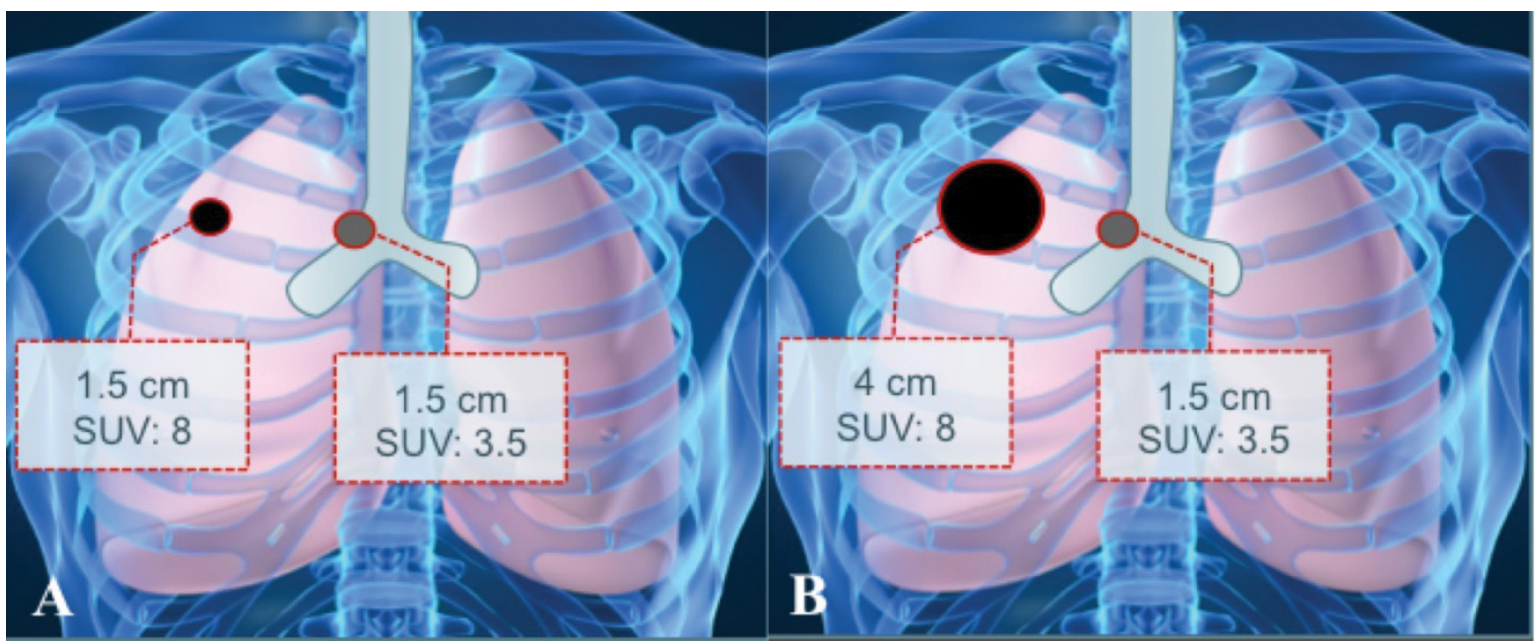

Figure 9: Figure showing a small RUL nodule with SUV differentiation.

should draw from both imaging modalities to relate uptake for size of nodes to that of the primary tumor. It also requires an understanding of lymphatic drainage pathways for lung cancer based on the location of the primary tumor.

\section{References}

1. Edge SB, Compton CC (2010) The American Joint Committee on Cancer: the 7th Edition of the AJCC Cancer Staging Manual and the Future of TNM. Ann Surg Oncol 17: 1471

2. Howington J, Blum MG, Chang AC, Balekian AA, Murthy SC (2013) Treatment of stage I and II non-small cell lung cancer: Diagnosis and management of lung cancer, 3rd ed: American College of Chest Physicians evidence-based clinical practice guidelines. Chest 143: e278S-e313S.

3. Tsim S, O'Dowd CA, Milroy R, Davidson S (2010) Staging of non-small cell lung cancer (NSCLC): A review. Respir Med 104: 1767-1774.

4. Billé A, Pelosi E, Skanjeti A, Arena V, Errico L (2009) Preoperative intrathoracic lymph node staging in patients with non-small-cell lung cancer: accuracy of integrated positron emission tomography and computed tomography Eur $\mathrm{J}$ Cardiothorac Surg 36: 440-445.

5. An YS, Sun JS, Park KJ, Hwang SC, Park KJ, et al. (2008) Diagnostic Performance of 18F-FDG PET/CT for Lymph Node Staging in Patients with Operable Non-small-cell Lung Cancer and Inflammatory Lung Disease, Lung (2008) 186: 327.
6. Gould MK, Kuschner WG, Rydzak CE, Maclean CC, Demas AN, et al (2003) Test Performance of Positron Emission Tomography and Computed Tomography for Mediastinal Staging in Patients with Non-Small-Cell Lung Cancer: A Meta-Analysis. Ann Intern Med. 139: 879-892.

7. Shim SS, Lee KS, Kim BT, Chung MJ, Lee EJ, et al. (2005) Non-Small Cell Lung Cancer: Prospective Comparison of Integrated FDG PET/CT and CT Alone for Preoperative Staging. Radiology 236: 1011-1019.

8. Steinert HC, Hauser M, Alleman F, Engel H, Berthold T, et al. (1997) Non-small cell lung cancer: nodal staging with FDG PET versus CT with correlative lymph node mapping and sampling. Radiology 202: 441-446.

9. Hellwig D, Graeter TP, Ukena D, Groeschel A, Sybrecht GW, et al. (2007) 18F-FDG PET for mediastinal staging of lung cancer: which SUV threshold makes sense? J Nucl Med 48: 1761-1766.

10. Nohl-Oser HC (1972) An investigation of the anatomy of the lymphatic drainage of the lungs as shown by the lymphatic spread of bronchial carcinoma. Ann R Coll Surg Engl 51: 157-176.

11. Fischer B, Lassen U, Mortensen J, Larsen S, Loft A, et al. (2009) Preoperative Staging of Lung Cancer with Combined PET-CT. N Engl J Med 361: 32-39.

12. Perigaud C, Bridji B, Roussel JC, Sagan C, Mugniot A, et al. (2009) Prospective preoperative mediastinal lymph node staging by integrated positron emission tomography-computerised tomography in patients with non-small-cell lung cancer. Eur J Cardiothorac Surg 36: 731-736. 